\section{Case Reports in Ophthalmology}

\title{
Wheel Spokes Technique for Endothelial Keratoplasty for Extremely Mydriatic Bullous Keratopathy Eyes without Capsular Support
}

\author{
Hidenori Tanaka $^{a} \quad K$ ji Hirano ${ }^{b} \quad$ Masayuki Horiguchi $^{a}$ \\ aDepartment of Ophthalmology, Fujita Health University School of Medicine, \\ Toyoake, Japan; 'bepartment of Ophthalmology, Ban Buntane Hospital, School of \\ Medicine, Nagoya, Japan
}

\section{Keywords}

Extremely mydriatic eye $\cdot$ Aphakic bullous keratopathy $\cdot$ Non-Descemet stripping automated endothelial keratoplasty · Wheel spokes technique

\begin{abstract}
We describe a new technique to prevent an endothelial donor graft from dropping into the vitreous cavity during non-Descemet stripping automated endothelial keratoplasty (nDSAEK) for extremely mydriatic bullous keratopathy (BK) eyes without capsular support. The patient was a 79-year-old woman who underwent nDSAEK for an extremely mydriatic BK eye with an incomplete barrier between the anterior and posterior chambers. She had undergone argon laser iridotomy for acute glaucoma at the age of 59 years and cataract surgery 3 years later. The pupil was extremely mydriatic as a result of iris sphincter muscle damage associated with an acute glaucoma attack and cataract surgery. After cataract surgery, the dislocated intraocular lens (IOL) sometimes touched the corneal endothelium. Despite simultaneous surgery to remove the dislocated IOL and lens capsule, vitrectomy, and intrascleral IOL fixation, her corneal endothelial decompensation progressed to BK. During nDSAEK, three 9-0 Prolene suture threads were placed through the recipient's cornea, limbus-to-limbus, resembling wheel spokes, to prevent the graft from dropping into the vitreous cavity. With the aid of these pre-
\end{abstract}


placed sutures, the graft was inserted safely and was well attached to the host's posterior cornea by air tamponade without suturing. The wheel spokes technique prevented the endothelial graft from dropping during intraoperative manipulation, suggesting that nDSAEK is possible even in an extremely mydriatic aphakic BK eye without capsule support.

(C) 2018 The Author(s)

Published by S. Karger AG, Basel

\section{Introduction}

Descemet stripping automated endothelial keratoplasty (DSAEK) has become one of the main surgical procedures to treat bullous keratopathy (BK). As it has become more mainstream, some reports have described the successful use of DSAEK for aniridic or extremely mydriatic aphakic BK without capsule support [1-4], which was previously considered difficult to treat. One reason for the challenge is that the barrier between the anterior and posterior chambers, such as the iris or lens capsule, is insufficient, so that the endothelial donor graft can drop from the anterior chamber into the vitreous cavity during intraoperative manipulation. Therefore, even if intraocular lens (IOL) suture fixation is performed prior to DSAEK, the inserted donor graft can still slip through the space between the IOL edge and iris into the vitreous cavity. In addition, air movement into the vitreous cavity could prevent sufficient maintenance of a tamponade effect, causing the graft to detach and even drop into the vitreous cavity. To prevent these complications, some groups have threaded a 10-0 Prolene suture through the edge of the donor graft, which was inserted into the anterior chamber (the so-called suture technique), and finally the suture was anchored onto the recipient cornea $[2,3]$.

Here, we report the successful use of non-DSAEK (nDSAEK) [5] for an extremely mydriatic aphakic BK eye with an incomplete barrier between the anterior and posterior chambers. We employed a simple technique to prevent donor graft dropping during surgery, using 3 limbus-to-limbus Prolene sutures in the cornea in a pattern resembling the spokes of a wheel. We termed it the "wheel spokes technique (WST) for endothelial keratoplasty."

\section{Case Report}

Our patient was a 79-year-old woman who previously underwent laser iridotomy for an acute glaucoma attack at the age of 59 years. Three years later, her cataracts had progressed, and she underwent phacoemulsification and IOL intracapsular fixation. The pupil was extremely mydriatic because of iris sphincter muscle damage associated with the acute glaucoma attack and previous cataract surgery. Corneal decompensation began to progress at the age of 79 years. This may have been partly attributable to laser iridotomy; in addition, a large part of Zinn's zonule had torn, causing the dislocated IOL to contact the corneal endothelium. We first extracted the IOL and then performed simultaneous vitrectomy and IOL intrascleral fixation. The intrascleral fixation employed a 3-piece foldable IOL with a diameter of $7.0 \mathrm{~mm}$ (EXTERNITY X-70, Santen Pharmaceutical Co., Ltd., Osaka, Japan), and 2 haptics were implanted at the 2 and 8 o'clock positions in the sclera, $2 \mathrm{~mm}$ posterior to the corneal limbus. This ultimately led to BK.

The patient required endothelial keratoplasty for an extremely mydriatic BK eye, after vitrectomy, with an incomplete barrier between the anterior and posterior chambers, despite IOL fixation. When DSAEK is performed in cases with an insufficient barrier between 
the anterior and posterior chambers, a piece of stripped Descemet membrane can fall into the vitreous cavity [1]; therefore, we elected to perform nDSAEK.

The surgery was performed under retrobulbar anesthesia [6] at Fujita Health University Hospital. The endothelial graft was cut with an 8.0-mm-diameter donor punch from the endothelial side. To prevent the graft from dropping backwards during insertion or intraoperative manipulation, a 9-0 Prolene suture attached to a straight needle and designed for ciliary sulcus fixation (Mani, Tochigi, Japan) was radially passed through the cornea, limbus-tolimbus, in a pattern resembling wheel spokes in the 3 and 9 o'clock, 6 and 12 o'clock, and 4 and 10 o'clock directions (Fig. 1a).

The endothelial graft was inserted into the patient's anterior chamber through a 5.1-mm temporal corneal tunnel using Kobayashi's double glide insertion technique [7]. The preplaced Prolene threads acted as a safety net to prevent the graft from falling into the vitreous cavity (Fig. 1b). After the corneal tunnel was sutured using 10-0 nylon, the graft was unfolded and centered by gently stroking the epithelial surface with a strabismus hook. After filling the anterior chamber with air (but not excessive air, as it might migrate into the vitreous cavity) for a tamponade to retain the graft, the 3 Prolene sutures were removed.

The interface fluid was drained from the 4 venting incisions of the patient's cornea under observation with microscope-integrated optic coherence tomography (OCT) (Carl Zeiss Rescan 700 ${ }^{\circledR}$; Carl Zeiss Meditec, Oberkochen, Germany).

Postoperatively, the patient was placed in a rigid supine position for $3 \mathrm{~h}$. Endothelial graft adherence was extremely favorable from the day after surgery.

\section{Discussion}

The WST is a simple and effective method to prevent the graft from dropping during nDSAEK and can be used not only for extremely mydriatic eyes but even for eyes with a total defect of the iris, including aniridia, whereas reconstruction of the barrier between the anterior and posterior chambers via pupilloplasty is not possible in such cases. The sutures stretched in a wheel spoke shape support the graft.

Our technique makes it possible to use Kobayashi's double glide donor insertion technique, which is useful in Asians with shallow anterior chambers. Furthermore, it avoids some troubles related to the so-called suture technique (e.g., endothelial damage associated with passing a suture through the graft, suture tangling, and graft inversion during insertion).

Theoretically, this approach requires 3 or more sutures from one side of the corneal limbus to the opposite side. Three equally spaced sutures produce a regular hexagon with 6 points, and 6 equilateral triangles are within that hexagon. Using an anterior segmental OCT (CASIA ${ }^{\circledR}$, Tomey Corp., Nagoya, Japan), the angle-to-angle distance in this patient was measured as $11.15 \mathrm{~mm}$. Therefore, the length of 1 side of the equilateral triangle is $5.58 \mathrm{~mm}$ (11.15 divided by 2). Even if an 8.0-mm diameter endothelial donor graft is unfolded (inserted into the anterior chamber, slightly bent by the Busin glide ${ }^{\circledR}$ ), the floating donor graft will not drop into the vitreous cavity unless the pulling forceps are instantly released (Fig. 2). In addition, the IOL had already been fixed in this case, so that the 2 haptics are considered to have an effect approximately equivalent to that of a single suture.

In the present case, it was not necessary to leave the anchored suture on the recipientdonor cornea after the operation. We intraoperatively confirmed that the interface fluid could be drained from the venting incision under microscope-integrated OCT observation, so 
the likelihood of postoperative graft detachment was deemed to be low. However, the limitation of WST is that it is only effective during graft insertion or intraoperative manipulation. If there are suspicions that graft detachment could occur postoperatively because the tamponade effect cannot be achieved fully when air moves into the vitreous cavity or because it is not possible to sufficiently drain the interface fluid, there should be no hesitation to put in an anchored suture.

\section{Conclusion}

In conclusion, the WST can be safely used to avoid endothelial graft dropping during nDSAEK even for extremely mydriatic aphakic BK eyes without capsular support.

\section{Statement of Ethics}

Written informed consent was obtained from the patient for the publication of this report.

\section{Disclosure Statement}

The authors have no conflicts of interest to disclose.

\section{References}

1 Price MO, Price FW Jr, Trespalacios R: Endothelial keratoplasty technique for aniridic aphakic eyes. J Cataract Refract Surg 2007;33:376-379.

2 Jastaneiah SS: Descemet's stripping-automated endothelial keratoplasty for traumatic aniridia and aphakia. Case Rep Ophthalmol Med 2012;2012:982657.

3 Patel AK, Luccarelli S, Ponzin D, Busin M: Transcorneal suture fixation of posterior lamellar grafts in eyes with minimal or absent iris-lens diaphragm. Am J Ophthalmol 2011;151:460-464.

4 Eguchi H, Miyamoto T, Hotta F, Tomida M, Inoue M, Mitamura Y: Descemet-stripping automated endothelial keratoplasty for vitrectomized cases with traumatic aniridia and aphakic bullous keratopathy. Clin Ophthalmol 2012;6:1513-1518.

5 Kobayashi A, Yokogawa H, Sugiyama K: Non-Descemet stripping automated endothelial keratoplasty for endothelial dysfunction secondary to argon laser iridotomy. Am J Ophthalmol 2008;146:543-549.

6 Sugimoto M, Horiguchi M, Tanikawa A, Shimada Y: Novel retrobulbar anesthesia technique through the sub-Tenon's space using a sharp needle in a blunt cannula. Ophthalmic Surg Lasers Imaging Retina 2013;44:483-486.

$7 \quad$ Kobayashi A, Yokogawa H, Sugiyama K: Descemet stripping with automated endothelial keratoplasty for bullous keratopathies secondary to argon laser iridotomy - preliminary results and usefulness of double-glide donor insertion technique. Cornea 2008;27(suppl 1):S62-S69. 

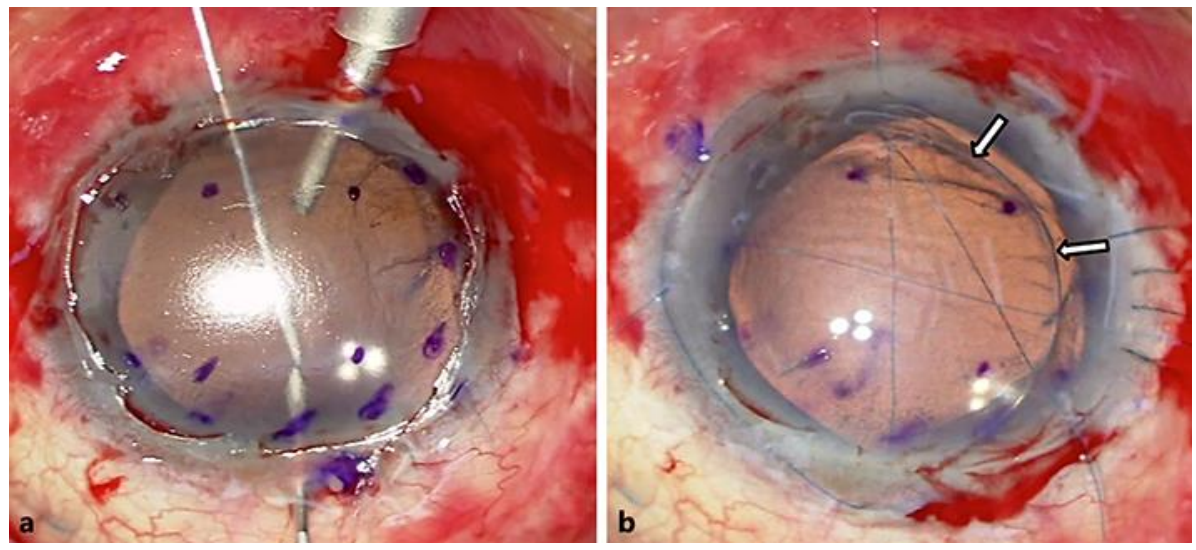

Fig. 1. Intraoperative photograph of setting the Prolene sutures for the wheel spokes technique. a Three straight needle-attached Prolene sutures were passed through the cornea in a limbus-to-limbus pattern resembling wheel spokes. $\mathbf{b}$ The 3 Prolene sutures support the floating donor graft (edge indicated by the arrows). Additionally, intraocular lens haptics were fixed to the sclera in the 2 o'clock-8 o'clock direction, posterior to the limbus, which could also serve as support.

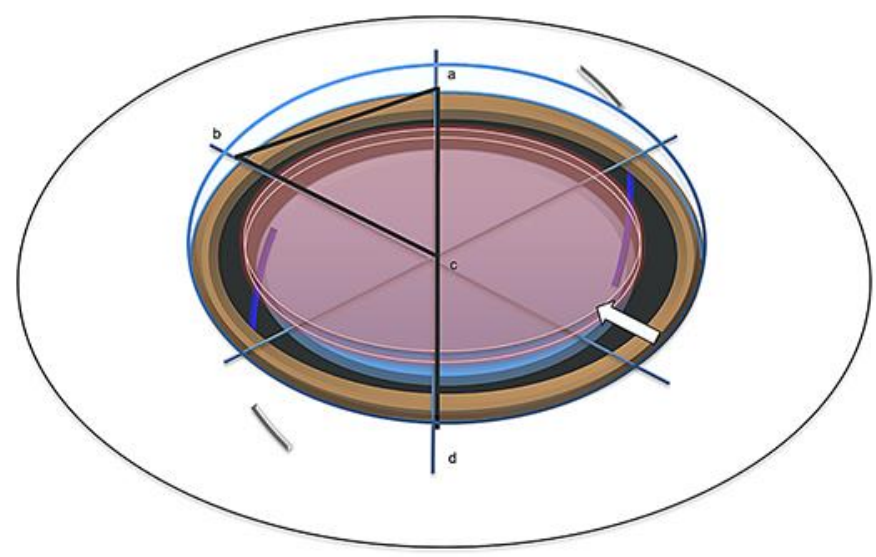

Fig. 2. Schematic illustration of the wheel spokes technique. The arrow indicates the inserted donor graft. An equilateral triangle defined by points $a, b$, and $c$ is formed by 3 threads that are evenly stretched across the corneal limbus. The distance a-d is $11.15 \mathrm{~mm}$, so $\mathrm{a}-\mathrm{b}$ is $5.58 \mathrm{~mm}$. Even if a donor graft with a diameter of $8.0 \mathrm{~mm}$ is inserted into the anterior chamber in a slightly folded shape using a Busin glide ${ }^{\circledR}$, the unfolded floating donor graft will not drop or slip into the vitreous cavity. 